\title{
Effects of drought on the microtranscriptome of field-grown sugarcane plants
}

\author{
Agustina Gentile • Thaís H. Ferreira • Raphael S. Mattos • Lara I. Dias • \\ Andrea A. Hoshino • Monalisa S. Carneiro • Glaucia M. Souza - Tercílio Calsa Jr. • \\ Rejane M. Nogueira $\cdot$ Laurício Endres $\cdot$ Marcelo Menossi
}

Received: 31 August 2012/Accepted: 21 October 2012/Published online: 6 November 2012

(C) The Author(s) 2012. This article is published with open access at Springerlink.com

\begin{abstract}
Sugarcane (Saccharum spp.) is the most promising crop for renewable energy. Among the diverse stresses that affect plant productivity, drought stress frequently causes losses in sugarcane fields. Although several studies have addressed plant responses to drought using controlled environments, plant responses under field conditions are largely unknown. Recently, microRNA
\end{abstract}

A. Gentile and T. H. Ferreira contributed equally to this work.

Electronic supplementary material The online version of this article (doi:10.1007/s00425-012-1795-7) contains supplementary material, which is available to authorized users.

A. Gentile $\cdot$ T. H. Ferreira - R. S. Mattos

L. I. Dias · A. A. Hoshino · M. Menossi ( $\square)$

Laboratório de Genoma Funcional, Departamento de Genética,

Evolução e Bioagentes, Instituto de Biologia, Universidade

Estadual de Campinas, Campinas, SP, Brazil

e-mail: menossi@unicamp.br

\section{S. Carneiro}

Departamento de Biotecnologia Vegetal, Centro de Ciências

Agrárias, Universidade Federal de São Carlos, Araras, SP, Brazil

\section{G. M. Souza}

Departamento de Bioquímica, Instituto de Química,

Universidade de São Paulo, São Paulo, Brazil

T. Calsa Jr.

Laboratório de Genômica e Proteômica de Plantas,

Departamento de Genética, Centro de Ciências Biológicas,

Universidade Federal de Pernambuco, Recife, PE, Brazil

R. M. Nogueira

Laboratório de Fisiologia Vegetal, Departamento de Biologia,

Universidade Federal Rural de Pernambuco, Recife, PE, Brazil

L. Endres

Centro de Ciências Agrárias, Universidade Federal de Alagoas,

Rio Largo, AL, Brazil
(miRNA)-mediated post-transcriptional regulation has been described as an important and decisive component in vegetal development and stress resistance modulation. The role of miRNAs in sugarcane responses to drought under field conditions is currently not known. Two sugarcane cultivars differing in drought tolerance were grown in the field with and without irrigation (rainfed) for 7 months. By using small RNA deep sequencing, we were able to identify 18 miRNA families comprising 30 mature miRNA sequences. Among these families, we found 13 mature miRNAs that were differentially expressed in droughtstressed plants. Seven miRNAs were differentially expressed in both cultivars. The target genes for many of the differentially expressed mature miRNAs were predicted, and some of them were validated by quantitative reverse transcription PCR. Among the targets, we found transcription factors, transporters, proteins associated with senescence, and proteins involved with flower development. All of these data increase our understanding of the role of miRNAs in the complex regulation of drought stress in field-grown sugarcane, providing valuable tools to develop new sugarcane cultivars tolerant to drought stress.

Keywords MicroRNAs - Bioenergy - Sugarcane · Drought stress · Field conditions · Solexa sequencing

\section{Introduction}

Sugarcane (Saccharum spp.) is a source of sugar and ethanol. This biofuel has been increasingly acknowledged as the most promising energy substitute for oil. Apart from being very productive, sugarcane is largely affected by biotic and abiotic stresses that lead to decreased yields (Boyer 1982; Maybank et al. 1995). Drought stress is one 
of the most important stresses that affect crops in many areas in the world. Under severe conditions, drought can produce irreversible alterations that could induce plant death. Drought causes several changes in sugarcane, such as the inhibition of root development, reduction in water and nutrient uptake, the decrease of leaf and stalk elongation, and in some cultivars, leaf rolling, which interferes with light absorption, reducing photosynthesis (InmanBamber and Smith 2005).

MicroRNAs (miRNAs) are an extensive family of small RNAs with a unique biogenesis (Axtell 2008; Voinnet 2009). They are small (19-24 nucleotides, nt), endogenous, single-stranded non-coding RNA molecules that hybridize to target mRNA and direct site-specific cleavage or translational repression (Carrington and Ambros 2003; Kidner and Timmermans 2006). MicroRNAs have been described as regulatory non-coding RNAs in plants and animals (Bartel 2004; Carrington and Ambros 2003). In plants such as Arabidopsis, Brachypodium, rice and maize, several studies have described miRNA genes as well as their targets in a wide variety of tissues, developmental stages, and treatment conditions (Gustafson et al. 2005; Jones-Rhoades and Bartel 2004; Rhoades et al. 2002; Unver and Budak 2009; Wu et al. 2009; Zhang et al. 2009a, b).

Because of their ability to regulate gene expression, many studies have focused on miRNAs. Plant miRNAs are often identical across large evolutionary distances (Axtell and Bartel 2005; Floyd and Bowman 2004) and are highly complementary to their targets, and this complementarity can be used to identify them using bioinformatics approaches (Fahlgren and Carrington 2010; Reinhart et al. 2002; Rhoades et al. 2002). Many predicted miRNA target genes encode regulatory proteins, suggesting that they function as important regulators (Bartel 2004). In Arabidopsis, $68 \%$ of the predicted conserved targets encode transcription factors that appeared to be involved in developmental patterning or stem cell identity (JonesRhoades et al. 2006). The same was reported in Brachypodium distachyon, where the majority of the predicted target genes encode transcription factors regulating plant development, morphology and flowering time (Unver and Budak 2009). Some reports revealed miRNA involvement in gene regulation under drought stress in rice (Zhao et al. 2007b) and maize (Zhang et al. 2009b). Because of the differences between animal and plant miRNAs in biogenesis, targets and mode of repression, it has been suggested that they originated independently in each kingdom (Axtell 2008). However, recent observations in the unicellular green algae Chlamydomonas reinhardtii support a more complex evolution (Molnar et al. 2007; Zhao et al. 2007c).

MicroRNA expression studies were greatly facilitated by the improvement of large-scale sequencing technologies that have been used in many studies (Pantaleo et al. 2010;
Ruby et al. 2006; Wei et al. 2009; Zhang et al. 2009a; Zhao et al. 2010). The Solexa technique allows for low-cost, high-quality and robust parallel sequencing of millions of 36 base-long fragments (Bentley 2006; Shendure et al. 2005). This methodology allows us to assess the expression profile of miRNAs by digital gene expression tag profiling (DGE). It is assumed that the number of times a particular sequence is observed in a cDNA sequencing library indicates the amount of that transcript in the sample. Through basic statistical tests, it is possible to compare the expression profiles of two samples. Recently, Hoen et al. (2008) obtained evidence that DGE detects more expression differences with fewer false-positives than quantitative realtime PCR and microarrays. To date, the majority of known plant miRNA sequences belong to Arabidopsis thaliana, Oryza sativa, and Populus trichocarpa because of their sequenced genomes. In this study, two field-grown sugarcane cultivars showing different responses to drought stress were analyzed. To the best of our knowledge, this is the first study involving sugarcane plants grown under field conditions and submitted to drought stress. The differentially expressed miRNAs were identified by high-throughput sequencing, and the miRNA targets were predicted in silico. Some of the targets were validated by quantitative reverse transcription PCR (RT-qPCR).

\section{Materials and methods}

\section{Plant samples}

Sugarcane cultivars RB867515 (high tolerance to drought, HT) and RB855536 (lower drought tolerance, LT) from RIDESA (Rede Interuniversitária para o Desenvolvimento do Setor Sucroenergetico) were fieldgrown in Campo Alegre, Alagoas, Brazil $\left(9^{\circ} 45^{\prime} 32^{\prime \prime} \mathrm{S}\right.$, $36^{\circ} 13^{\prime} 09^{\prime \prime} \mathrm{W}$ ), and samples were collected on 04 April 2009, 7 months under irrigation or without irrigation (rainfed). The cultivars RB867515 (RB72454 $\times * * * * *$ ?) and RB855536 (SP70-1143 $\times$ RB72454) are derived from half-sib families and exhibit different responses to water deficit. The root system of the LT cultivar (RB855536) is less developed in deeper soil layers (20-80 cm) (Santos 2010; Vasconcelos et al. 2003). The length/root mass ratio is one of the RB855536 phenotypic features that indicates the lower tolerance to drought. The roots from the cultivar HT (RB867515) shows a homogeneous distribution throughout the layers of the soil profile $(0-80 \mathrm{~cm})$, favoring a higher tolerance to drought (Santos 2010). Sugarcane plants were grown at field conditions during the dry season (Supplementary Fig. S1). Irrigated plots received $60 \mathrm{~mm}$ of irrigation every month. In irrigated plants, samples were collected 
5 days after applying water to the fields, ensuring that plants were well watered. Rainfed plants experienced water deficit throughout the 7-month period, except in February 2009, when plant rainfall matched plant water demands (Supplementary Fig. S1). At the time point plant samples were collected, plants were experiencing a water deficit (Supplementary Fig. S1) and the last rain occurred 15 days before (data not shown). Several physiological parameters evidenced that rainfed plants were under drought stress (Supplementary Fig. S1). Leaf +1 tissue (the highest expanded leaf with a visible dewlap) was collected in quadruplicate after 7 months from irrigated and rainfed drought-stressed plants. Samples were snap-frozen and maintained at $-80{ }^{\circ} \mathrm{C}$. Two replicates were combined and used for Solexa sequencing.

\section{Leaf total RNA extraction}

Total RNA was isolated using the miRVana ${ }^{\mathrm{TM}}$ miRNA isolation kit (Life Technologies, USA) according to manufacturer's protocol, with minor modifications. Briefly, ten volumes of lysis/binding buffer per macerated leaf-tissue mass were added into a tube and mixed. One volume of miRNA homogenate was added to the tissue lysate and mixed by vortexing. After $10 \mathrm{~min}$ on ice, ten volumes of acid-phenol:chloroform were added and mixed gently. The samples were then centrifuged for $7 \mathrm{~min}$ at $10,000 \times g$ at room temperature to separate the aqueous and organic phases. The aqueous phase was removed carefully and transferred to a new tube. A $1.25 \times$ volume of absolute ethanol was added to the aqueous phase, mixed, and placed onto the filter cartridge. Samples were centrifuged $20 \mathrm{~s}$ at $10,000 \times g$ to pass the mixture through the filter. The samples on the filters were then washed, the filter transferred to a new tube and the RNA eluted in $80 \mu \mathrm{L}$ of preheated nuclease-free water. Total RNA samples were quantified (NanoDrop, Thermo Scientific, USA) and stored at $-80{ }^{\circ} \mathrm{C}$ for later use.

\section{Small RNA sequencing}

The cDNA library synthesis and sequencing were performed at BGI (Beijing Genomic Institute, Tai Po, Hong Kong) using the Solexa platform. Briefly, total RNA samples received at the company were analyzed in a 2100 Bioanalyzer (Agilent, USA) to check for integrity and quality. Before constructing the miRNA libraries, RNAs from 16 to 27 nucleotides long were selected by polyacrylamide gel electrophoresis, ligated with adaptors at both ends and the products used for cDNA synthesis. Then, they were PCR-amplified and sequenced using the Solexa technology.
Bioinformatics analysis

The prediction of the sugarcane precursors (pre-miRNAs) was performed by searching for sequences that matched with the validated mature miRNAs. The adaptors were then removed, and any reads shorter than 19 nucleotides or longer than 24 nucleotides were discarded. The raw data with all sequences used in this work may be available upon request. The transcripts were mapped to the Sorghum bicolor genome and sugarcane transcriptome as references using the miRDeep-P program (Yang and Li 2011). For a given mapped read, the optimal window size was $250 \mathrm{bp}$, which was used to extract reference sequences for predicting the RNA secondary structure (Yang et al. 2011). The miRDeep core algorithm with a plant-specific scoring system based on the known characteristics of plant miRNA genes was used to find the secondary structures of the sequences (Meyers et al. 2008). RNA sequences were considered miRNA precursor candidates if the following conditions were met: the RNA sequence could fold into the characteristic stem-loop hairpin secondary structure, the mature miRNA lays within one arm of the hairpin structure and had a maximum of six mismatches with the miRNA* sequence in the opposite arm, the predicted secondary structures had negative MFEs, and the G/C content was between 30 and $70 \%$ (Zanca et al. 2010). All the secondary structures of the precursors were predicted using the RNAfold program (Hofacker 2003).

After the normalization of the number of reads, the expression of each miRNA was calculated based on the Audic-Claverie method (Audic and Claverie 1997). The target of each miRNA was predicted by psRNATarget (http://plantgrn.noble.org/psRNATarget/), which searches for target genes based on complementarity scoring and secondary structure analysis (Dai and Zhao 2011).

\section{Evaluation of miRNA and miRNA targets expression profiles by RT-qPCR}

RT-loop primers (loop-RT), forward specific PCR primers (loop-FW) and reverse universal primers were designed following Chen et al. (2005) (Supplementary Table S1) for reverse transcription and PCR amplification of sugarcane miRNAs, of two sugarcane genes related to drought stress, encoding DREB and dehydrin homologs, and to validate some of the target genes. Reverse transcriptase reactions were performed as described by Varkonyi-Gasic et al. (2007). Each reaction contained $2.5 \mu \mathrm{g}$ of DNA-free total RNA, $1 \mu \mathrm{L}$ of each RT-loop primer $(1 \mu \mathrm{M}), 1 \mu \mathrm{L}$ oligo $\mathrm{d}(\mathrm{T})_{17} \mathrm{VN}(50 \mu \mathrm{M})$, and $1 \mu \mathrm{L}$ of dNTP mix $(10 \mu \mathrm{M})$. The reaction was incubated for $10 \mathrm{~min}$ at $65{ }^{\circ} \mathrm{C}$ and then placed on ice for $2 \mathrm{~min}$. Subsequently, 5X First Strand Buffer, DTT, RNAseOut, and Superscript III enzyme (Life 
Technologies, USA) were added. This reaction was incubated in a Verity ${ }^{\mathrm{TM}}$ Thermal Cycler (Applied Biosystems, USA) for $30 \mathrm{~min}$ at $16{ }^{\circ} \mathrm{C}$, followed by 60 cycles of $30{ }^{\circ} \mathrm{C}$ for $30 \mathrm{~s}, 42^{\circ} \mathrm{C}$ for $30 \mathrm{~s}$, and $50{ }^{\circ} \mathrm{C}$ for $1 \mathrm{~s}$. Finally, the reaction was incubated $5 \mathrm{~min}$ at $85{ }^{\circ} \mathrm{C}$ for the enzyme inactivation.

Real-time PCR was performed to analyze the expression of sugarcane genes. The reactions were carried out using the SYBR Green PCR Master Mix (Applied Biosystems, USA) on 7500 Real-Time PCR System (Applied Biosystems, USA). Each $18 \mu \mathrm{L}$ PCR reaction included $2 \mu \mathrm{L}$ of cDNA, $10 \mu \mathrm{L}$ of SYBR Green Master Mix $(1 \times), 1 \mu \mathrm{L}$ of forward primer $(10 \mu \mathrm{M}), 1 \mu \mathrm{L}$ of reverse primer $(10 \mu \mathrm{M})$ (Supplementary Table S1), and water. The polyubiquitin gene (Papini-Terzi et al. 2005) was used as a reference. The reactions were performed at $95{ }^{\circ} \mathrm{C}$ for $10 \mathrm{~min}$, followed by 40 cycles of $95^{\circ} \mathrm{C}$ for $15 \mathrm{~s}$ and $60^{\circ} \mathrm{C}$ for $1 \mathrm{~min}$ with a final dissociation curve analysis. All reactions were run in triplicate with three biological replicates.

The real-time PCR data analysis was performed based on the reaction efficiencies required to calculate the foldchanges and using the web-based QPCR system (Pabinger et al. 2009).

\section{Results}

Physiological data and confirmation of drought stress in sugarcane plants

Plants from the cultivars RB867515 (HT to drought) and RB855536 (LT to drought) were grown in the field for
7 months with and without irrigation (rainfed). Water deficit negatively affected the photosynthetic activity and reduced the stomatal conductance (gs), photosynthesis (A), transpiration rate $(\mathrm{E})$, and water and osmotic potentials $\left(\Psi_{\mathrm{w}}\right.$ and $\Psi_{\mathrm{o}}$ ), indicating a reduction in the photosynthetic performance (Supplementary Fig. S1). For all of the analyzed parameters, the HT cultivar was less affected by drought stress, demonstrating a better response under adverse conditions (Supplementary Fig. S1).

To further confirm that plants were stressed, we evaluated the expression of a sugarcane gene encoding a dehydrin (Sugarcane Assembled Sequence, SAS: SCQGLR 1085F11.g) by RT-qPCR (Fig. 1). This gene was already described as drought-induced in sugarcane (Rocha et al. 2007). A second gene encoding a homolog of a DREB transcription factor (SAS: SCJLLR2013H07.g) was also used. This class of transcription factor was reported to be induced by cold and dehydration in plants (Agarwal et al. 2006). In sugarcane plants, the dehydrin gene was induced in both cultivars and showed higher levels in the HT cultivar RB867515 (Fig. 1). Interestingly, the DREB homolog was upregulated only in the HT cultivar (Fig. 1). As expected, these data show that drought was affecting the sugarcane transcriptome.

\section{Identification of sugarcane miRNAs under water deficit}

To identify miRNAs from Saccharum spp., a highthroughput approach using Solexa sequencing was used, and the sequences were compared to the miRBase database. To this end, total RNA samples were obtained from the mature leaves of two different sugarcane cultivars,

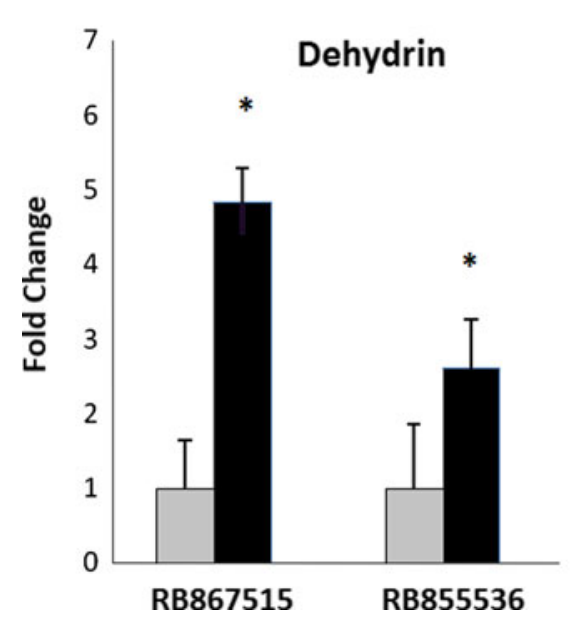

Fig. 1 RT-qPCR of two sugarcane genes encoding dehydrin (SCQGLR1085F11.g) and the DREB transcription factor (SAS: SCJLLR2013H07.g). RB867515 (higher drought tolerance, HT) and RB855536 (lower drought tolerance, LT) plants were irrigated (grey bars) or subjected to water deficiency by withholding irrigation (black

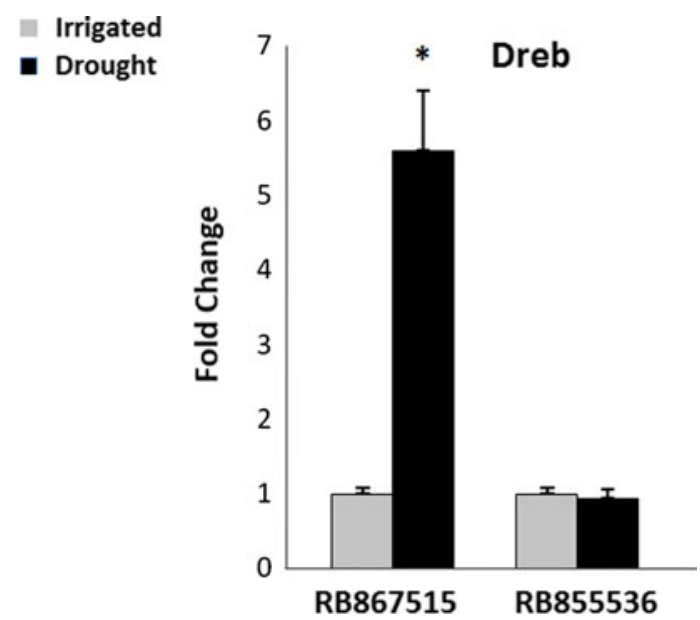

bars) for 7 months. RB867515-irrigated was used as reference sample to calculate the fold change. Error bars represent the standard error $(n=2), * p<0.05$. Statistics were calculated between irrigated and non-irrigated treatments in each cultivar using a permutation test. The expression in irrigated RB867515 plants was considered as 1 
Table 1 Small RNA deep-sequencing results for sugarcane leaves from RB867515 (HT) and RB855536 (LT) cultivars under irrigation (I) and drought (D) conditions after 7 months of stress on the field

\begin{tabular}{|c|c|c|c|c|c|c|c|c|}
\hline \multirow[t]{2}{*}{ Category } & \multicolumn{4}{|l|}{ HTI } & \multicolumn{4}{|l|}{ HTD } \\
\hline & Unique RNAs & Percent $(\%)$ & Total RNAs & Percent $(\%)$ & Unique RNAs & Percent (\%) & Total RNAs & Percent $(\%)$ \\
\hline miRNA & 33,295 & 0.86 & $2,568,341$ & 21.04 & 15,074 & 0.71 & $1,547,690$ & 20.47 \\
\hline rRNA & 57,826 & 1.49 & 690,977 & 5.66 & 80,838 & 3.8 & $1,307,606$ & 17.29 \\
\hline siRNA & 99,469 & 2.57 & 640,673 & 5.24 & 31,807 & 1.49 & 208,823 & 2.76 \\
\hline snRNA & 2,778 & 0.07 & 13,047 & 01 & 2,893 & 0.14 & 14,372 & 0.19 \\
\hline snoRNA & 1,457 & 0.04 & 4,727 & 0.04 & 1,05 & 0.05 & 3,2938 & 0.04 \\
\hline tRNA & 15,783 & 0.41 & $1,058,986$ & 8.67 & 17,416 & 0.82 & 479,527 & 6.34 \\
\hline Unannotated & $3,660,105$ & 94.55 & $7,230,626$ & 59.23 & $1,978,922$ & 92.99 & $4,000,025$ & 52.9 \\
\hline Total small RNAs & $3,870,713$ & 100.00 & $12,207,377$ & 100.00 & $2,128,000$ & 100.00 & $7,561,341$ & 100.00 \\
\hline \multirow[t]{2}{*}{ Category } & \multicolumn{4}{|l|}{ LTI } & \multicolumn{4}{|l|}{ LTD } \\
\hline & Unique RNAs & Percent $(\%)$ & Total RNAs & Percent $(\%)$ & Unique RNAs & Percent $(\%)$ & Total RNAs & Percent $(\%)$ \\
\hline miRNA & 15,663 & 0.52 & $1,294,640$ & 12.98 & 19,966 & 0.72 & $1,853,916$ & 17.76 \\
\hline rRNA & 108,411 & 3.57 & $1,994,571$ & 20.00 & 83,009 & 2.99 & $1,604,192$ & 14.41 \\
\hline siRNA & 55,071 & 1.81 & 402,188 & 4.03 & 44,402 & 1.6 & 255,345 & 2.45 \\
\hline snRNA & 4,955 & 0.16 & 43,655 & 0.44 & 3,797 & 0.14 & 2134 & 0.21 \\
\hline snoRNA & 2,184 & 0.07 & 10,697 & 0.11 & 2,624 & 0.09 & 18,403 & 0.18 \\
\hline tRNA & 23,831 & 0.78 & 766,089 & 7.68 & 19,327 & 0.7 & 547,433 & 5.25 \\
\hline Unannotated & $2,825,979$ & 93.08 & $5,461,047$ & 54.76 & $2,605,783$ & 93.77 & $6,235,247$ & 59.75 \\
\hline Total small RNAs & 3.036 .094 & 100.00 & $9,972,887$ & 100.00 & $2,778,908$ & 100.00 & $10,436,376$ & 100.00 \\
\hline
\end{tabular}

RB867515 (HT) and RB855536 (LT), that were grown in the field for 7 months with (I) or without (D) irrigation. These four cDNA libraries yielded between 8 and 12 million clean reads each (Table 1) and more than 40 million reads in total. Good-quality reads between 18 and 25 bases were analyzed. In both cultivars under either condition (irrigated and stressed), the most abundant sRNAs were either 21-nt or 24-nt long, representing miRNAs and siRNAs, respectively (Fig. 2). This pattern was also observed in sequencing analyses from other plant species (Chapman and Carrington 2007). After annotation of the unique tags using the RFam and GenBank RNA databases, the remaining tags were compared to sorghum miRNAs in miRBase, which resulted in 18 families and 30 mature miRNA sequences (Table 2).

Bioinformatics identification of sugarcane miRNA precursors

The whole set of miRNA sequences shown in Table 2 was mapped onto SoGI and SUCEST databases to identify their precursors. In total, eight precursors corresponding to seven miRNA families were found, and these corresponded to precursors already deposited in miRBase (Ferreira et al. 2012; Zanca et al. 2010). Seven precursor sequences were from the SUCEST (http://sucest-fun.org/) database, whereas only one was found in the SoGI (http://compbio.dfci. harvard.edu/) database (data not shown). Two additional miRNA precursors (ssp-MIR168 and ssp-MIR396) were found in comparison with our previous work with sugarcane plants grown in glasshouses (Ferreira et al. 2012). All of the precursor sequences found in both databases have the capacity to fold into hairpin structures and hold the mature miRNA in one arm of the hairpin structure, which, together with the negative MFEs energy values and the G/C content, supports the veracity of the sugarcane precursors (Ferreira et al. 2012; Zanca et al. 2010).

Differential expression of sugarcane miRNAs under drought stress

We have identified 13 differentially expressed mature miRNAs, using a $p$ value $<0.05$ and fold change $\geq 2$ (Table 3). The HT cultivar had 11 miRNAs that were differentially expressed between the irrigated and droughtstressed plants (HTI $\times$ HTD), while the LT cultivar had nine miRNAs modulated by drought stress (LTI $\times$ LTD). Among the 11 miRNAs found in the HT cultivar, 3 were upregulated (ssp-miR160-seq 3, ssp-miR399-seq 3, and ssp-miR528), and 8 were downregulated (ssp-miR166-seq 3, ssp-miR169-seq 2, ssp-miR171-seq 2, ssp-miR172, sspmiR393, ssp-miR396, ssp-miR399-seq 2, and ssp-miR1432). 


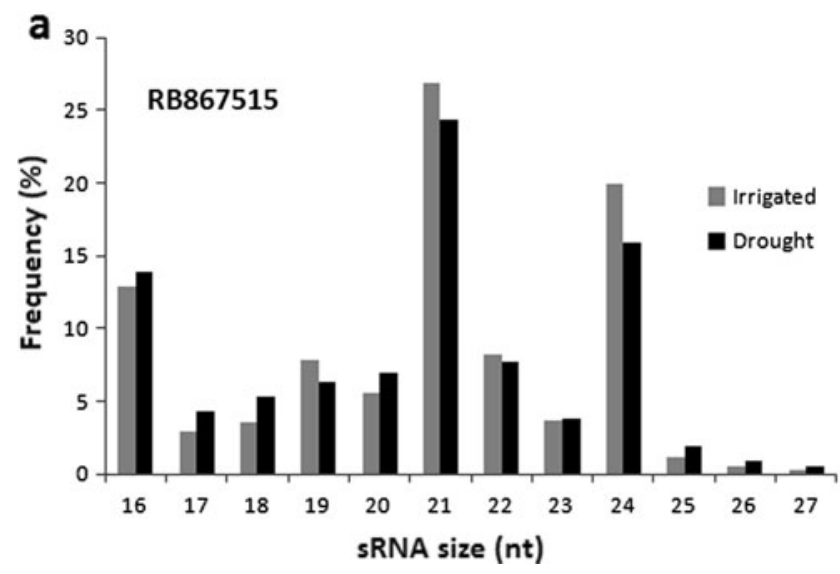

Fig. 2 Size distribution of small RNA (sRNA) sequences in sugarcane. Plants of two cultivars, RB867515 (a) and RB855536 (b), were field-grown for 7 months in two conditions irrigated and drought-

In the LT cultivar, six were upregulated (ssp-miR160-seq 1, ssp-miR160-seq 3, ssp-miR394, ssp-miR399-seq 2, sspmiR399-seq 3, and ssp-miR1432), and three were downregulated (ssp-miR166-seq 3, ssp-miR171-seq 2, and ssp-miR396). Both cultivars shared seven differentially expressed miRNAs in this experiment: ssp-miR160-seq 3, spp-miR166-seq 3, ssp-miR171-seq 2, ssp-miR396, sspmiR399-seq 2, spp-miR399-seq 3, and ssp-miR1432. Among them, five were induced or repressed in both cultivars (sspmiR160-seq 3, spp-miR166-seq 3, ssp-miR171-seq 2, ssp-miR396, and spp-miR399-seq 3), while the other two (ssp-miR399-seq 2 and ssp-miR1432) had the opposite patterns (Table 3).

The sequencing results showed that ssp-miR160-seq 1 , ssp-miR160-seq 3, ssp-miR394, ssp-miR399-seq 2, sspmiR399-seq 3, ssp-miR528, and ssp-miR1432 were induced under drought stress compared to controls, in at least one cultivar (Fig. 3). The majority was induced only in the LT cultivar (RB855536), with the exception of sspmiR528, which was expressed more highly under drought in the HT cultivar (RB867515). Only ssp-miR160-seq 3 and ssp-miR399-seq 3 were induced under drought in both cultivars.

In contrast, some miRNAs were repressed under drought treatment, and most of them were downregulated in the HT cultivar. Only ssp-miR166-seq 3, ssp-miR171-seq 2, and ssp-miR396 were repressed under drought in both cultivars (Fig. 3).

The expression profiles of two miRNAs (ssp-miR160seq1 and ssp-miR528) modulated by drought were further analyzed by qRT-PCR. These miRNAs presented expression patterns that were similar to those observed with deepsequencing (Supplementary Fig. S2).

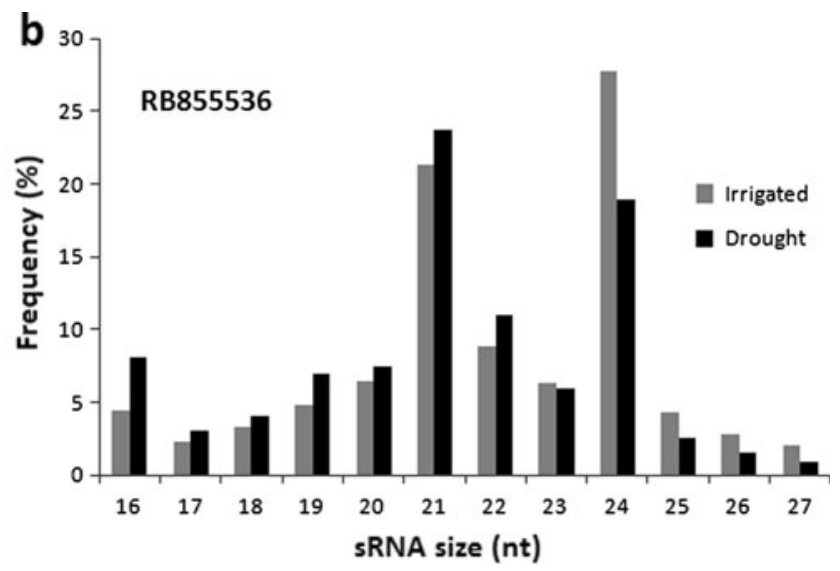

stressed. RB867515 is known as higher drought tolerant (HT) and RB855536 as lower drought tolerant (LT). The sRNA size is shown in number of nucleotides (nt)

Prediction of sugarcane miRNA targets

Many of the differentially expressed miRNAs had target candidates in the SUCEST database, with the exception being ssp-miR160-seq 3, ssp-miR396 and ssp-miR399-seq 2 (Table 4). Among the targets, most of them encode transcription factors (ssp-miR160-seq 1, ssp-miR166-seq 3, sspmiR169-seq 2, ssp-miR171-seq 2, ssp-miR172, ssp-miR528 and ssp-miR1432, among others). Other targets encode transporters (ssp-miR172, ssp-miR528, ssp-miR1432), proteins associated with senescence (ssp-miR399-seq 3) and proteins involved with flower development (ssp-miR172) (Table 4, Supplementary Table S2).

\section{Analysis of target gene expression}

To evaluate whether the miRNA expression profiles correlated with differences in the transcripts from the target genes, the expression of four targets corresponding to the four miRNAs with the highest expression (miR160-seq 1, miR172, miR528, and miR1432; Fig. 3) were evaluated by RT-qPCR (Fig. 4). To facilitate the comparison between the expression of miRNAs and their corresponding target genes (Figs. 3, 4), we calculated the ratios between drought-stressed and irrigated expression levels (Table 5). Among the eight expression profiles, six cases behaved as expected, i.e., miRNA expression was induced and target gene expression was repressed upon drought stress and vice versa (shown in bold in Table 5). In four out of five cases where the miRNA profiles had a $p$ value $<0.05$ (Fig. 3), the expected trend in the profile of the target gene was also significant at $p<0.05$. In two cases, the miRNA and target gene profiles showed no agreement in the LT cultivar 
Table 2 Sugarcane miRNA families and mature miRNAs identified by Solexa sequencing

\begin{tabular}{|c|c|c|c|}
\hline miR family & miRNA name & Mature sequences & Sorghum precursor \\
\hline \multirow[t]{7}{*}{$\operatorname{miR} 156$} & ssp-miR156-seq 1 & UUGACAGAAGAGAGUGAGCAC & sbi-MIR156a \\
\hline & & & sbi-MIR156b \\
\hline & & & sbi-MIR156c \\
\hline & ssp-miR156-seq 2 & UGACAGAAGAGAGCGAGCAC & sbi-MIR156e \\
\hline & ssp-miR160-seq 1 & UGCCUGGCUCCCUGUAUGCCA & sbi-MIR160c \\
\hline & & & sbi-MIR160a \\
\hline & & & sbi-MIR160d \\
\hline \multirow[t]{2}{*}{$\operatorname{miR} 160$} & ssp-miR160-seq 2 & UGCCUGGCUCCCUGAAUGCCA & sbi-MIR160f \\
\hline & ssp-miR160-seq 3 & AGGUAGAGGAGAAGAGUG & sbi-MIR160b \\
\hline $\operatorname{miR164}$ & ssp-miR164 & UGGAGAAGCAGGGCACGUGCU & sbi-MIR164b \\
\hline \multirow[t]{5}{*}{$\operatorname{miR} 166$} & ssp-miR166-seq 1 & UCGGACCAGGCUUCAUUCCCC & sbi-MIR166b \\
\hline & & & sbi-MIR166c \\
\hline & & & sbi-MIR166d \\
\hline & ssp-miR166-seq 2 & UCGGACCAGGCUUCAUUCCUC & sbi-MIR166f \\
\hline & & & sbi-MIR166k \\
\hline \multirow[t]{7}{*}{$\operatorname{miR} 167$} & ssp-miR166-seq 3 & UCGGACCAGGCUUCAAUCCCU & sbi-MIR166e \\
\hline & & & sbi-MIR166f \\
\hline & & & sbi-MIR166g \\
\hline & ssp-miR167b & UGAAGCUGCCAGCAUGAUCU & sbi-MIR167a \\
\hline & & UGAAGCUGCCAGCAUGAUCUGA & sbi-MIR167g \\
\hline & & & sbi-MIR167h \\
\hline & & UGAAGCUGCCAGCAUGAUCUG & sbi-MIR167c \\
\hline $\operatorname{miR} 168$ & ssp-miR168a & UCGCUUGGUGCAGAUCGGGAC & sbi-MIR168 \\
\hline \multirow[t]{2}{*}{$\operatorname{miR} 169$} & ssp-miR169-seq 1 & GGGCAAAUCAUCCGGGCUAGC & sbi-MIR169o \\
\hline & ssp-miR169-seq 2 & CGGCAAGUUGUUCUUGGCUAC & sbi-MIR169a \\
\hline \multirow[t]{2}{*}{$\operatorname{miR} 171$} & ssp-miR171-seq 1 & UUGAGCCGCGUCAAUAUCUCC & sbi-MIR171h \\
\hline & ssp-miR171-seq 2 & UGAUUGAGCCGUGCCAAUAUC & sbi-MIR171i \\
\hline $\operatorname{miR} 172$ & ssp-miR172 & AGAAUCUUGAUGAUGCUGCAU & sbi-MIR172d \\
\hline $\operatorname{miR} 393$ & ssp-miR393 & CUCCAAAGGGAUCGCAUUGAU & sbi-MIR393b \\
\hline $\operatorname{miR} 394$ & ssp-miR394 & UUGGCAUUCUGUCCACCUCC & sbi-MIR394b \\
\hline \multirow[t]{12}{*}{$\operatorname{miR} 395$} & spp-miR395-seq 1 & GUUCCCUGCAAGCACUUCACA & sbi-MIR395b \\
\hline & & & sbi-MIR395a \\
\hline & & & sbi-MIR395c \\
\hline & & & sbi-MIR395e \\
\hline & & & sbi-MIR395f \\
\hline & & & sbi-MIR395g \\
\hline & & & sbi-MIR395h \\
\hline & & & sbi-MIR395i \\
\hline & & & sbi-MIR395d \\
\hline & spp-miR395-seq 2 & UGAAGUGUUUGGGGGAACUC & sbi-MIR395i \\
\hline & & & sbi-MIR395j \\
\hline & & & sbi-MIR395k \\
\hline $\operatorname{miR} 396$ & ssp-miR396 & UUCCACAGCUUUCUUGAA & sbi-MIR396b \\
\hline $\operatorname{miR} 397$ & ssp-miR397 & UUGACUGCAGCGUUGAUGAGC & sbi-MIR397 \\
\hline
\end{tabular}


Table 2 continued

\begin{tabular}{llll}
\hline miR family & miRNA name & Mature sequences & Sorghum precursor \\
\hline miR399 & ssp-miR399-seq 1 & UGCCAAAGGAGAGUUGCCCU & sbi-MIR399i \\
& ssp-miR399-seq 2 & UGCCAAAGGAGAAUUGCCC & sbi-MIR399h \\
& & & sbi-MIR399j \\
& & & sbi-MIR399b \\
miR528 & ssp-miR399-seq 3 & GUGCAGCUCUCCUCUGGCAUG & sbi-MIR528 \\
miR529 & ssp-miR528 & UGGAAGGGGCAUGCAGAGGAG & sbi-MIR529 \\
miR1432 & ssp-miR529 & AGAAGAGAGAGAGUACAGCCU & sbi-MIR1432 \\
\hline
\end{tabular}

miRNAs were found in the leaves of two sugarcane cultivars, one with higher tolerance to drought (HT, RB867515) and the other with lower tolerance to drought (LT, RB855536). Two mismatches were allowed using sorghum mature miRNAs as references

Table 3 Differentially expressed mature microRNAs found under drought stress in sugarcane

\begin{tabular}{|c|c|c|c|c|c|c|c|c|}
\hline Family & miRNA name & Mature sequences & HTI (TPM) & HTD (TPM) & LTI (TPM) & LTD (TPM) & HTD/HTI & LTD/LTI \\
\hline \multirow{2}{*}{ miR160 } & ssp-miR160-seq 1 & UGCCUGGCUCCCUGUAUGCCA & 129.61 & 96.1 & 9.57 & 92.13 & -1.35 & 9.63 \\
\hline & ssp-miR160-seq 3 & AGGUAGAGGAGAAGAGUG & 1.1 & 2.41 & 0.74 & 1.59 & 2.19 & 2.15 \\
\hline miR166 & ssp-miR166-seq 3 & UCGGACCAGGCUUCAAUCCCU & 22.95 & 10.67 & 29.07 & 6.59 & -2.15 & -4.41 \\
\hline miR169 & ssp-miR169-seq 2 & CGGCAAGUUGUUCUUGGCUAC & 57.22 & 15.94 & 12.31 & 11.24 & -3.59 & -1.1 \\
\hline miR171 & ssp-miR171-seq 2 & UGAUUGAGCCGUGCCAAUAUC & 6.99 & 1.09 & 3.2 & 1.5 & -6.41 & -2.13 \\
\hline miR172 & ssp-miR172 & AGAAUCUUGAUGAUGCUGCAU & 3406.55 & 1325.44 & 924.09 & 687.42 & -2.57 & -1.34 \\
\hline miR393 & ssp-miR393 & CUCCAAAGGGAUCGCAUUGAU & 231.62 & 72.01 & 86.82 & 91.17 & -3.22 & 1.05 \\
\hline miR394 & ssp-miR394 & UUGGCAUUCUGUCCACCUCC & 3.03 & 1.57 & 0.1 & 1.24 & -1.93 & 12.4 \\
\hline miR396 & ssp-miR396 & UUCCACAGCUUUCUUGAA & 67.22 & 27.33 & 28.43 & 8.87 & -2.46 & -3.21 \\
\hline \multirow{2}{*}{ miR399 } & ssp-miR399-seq 2 & UGCCAAAGGAGAAUUGCCC & 1.79 & 0.73 & 0.1 & 0.71 & -2.45 & 7.1 \\
\hline & ssp-miR399-seq 3 & GUGCAGCUCUCCUCUGGCAUG & 1.1 & 2.41 & 0.74 & 22.29 & 2.19 & 30.12 \\
\hline miR528 & ssp-miR528 & UGGAAGGGGCAUGCAGAGGAG & 1217.07 & 29286.9 & 28721.93 & 51914.2 & 24.1 & 1.81 \\
\hline miR1432 & ssp-miR1432 & UCAGGAAAGAUGACACCAA & 6213.68 & 2475.84 & 245.94 & 730.5 & -2.51 & 2.97 \\
\hline
\end{tabular}

HT cultivar RB867515, LT cultivar RB855536. Green boxes indicate upregulated under drought, red boxes indicate downregulated under drought, considering a $p$ value $<0.05$ and fold change $\geq 2$. Statistics were calculated between irrigated and drought treatments in each cultivar using the Audic-Claverie method

$H T I$ irrigated higher tolerant, $H T D$ drought higher tolerant, $L T I$ irrigated lower tolerant, $L T D$ drought lower tolerant, $T P M$ transcripts per million

(Table 5), which suggests that the regulation by miRNA might be influenced by the genetic background.

\section{Discussion}

There are many recent reports confirming the identification of miRNAs in different plants, and some of these also describe differential expression patterns of miRNAs under stress (Jian et al. 2010; Jones-Rhoades and Bartel 2004; Matts et al. 2010; Pantaleo et al. 2010; Ruan et al. 2009; Unver and Budak 2009; Xin et al. 2010; Zhang et al. 2009b; Zhao et al. 2010; Zhou et al. 2010). Although a small number of sugarcane miRNAs have already been registered in miRBase, to the best of our knowledge, this is the first report that associates drought stress and miRNA identification in field-grown sugarcane cultivars based on sequencing profiles.

During the development of the Sugarcane Expressed Sequence Tag Project (SUCEST), 43,141 transcripts of sugarcane (SAS) were generated (Vettore et al. 2001). Approximately $30 \%$ of the SAS had no significant identity to sequences from other organisms and might be involved in the post-transcriptional regulation of other genes. Currently, there are only 34 sugarcane miRNA sequences predicted by bioinformatics in the miRBase database, 
Fig. 3 Expression profile based on the sequencing data of 13 differentially expressed sugarcane microRNAs. The value is expressed as the number of transcripts per million (TPM) for both conditions irrigated (control, grey bars) and drought-stressed (black bars) for the RB867515 (higher tolerance to drought) and RB855536 (lower tolerance to drought) cultivars. Each sample was a pool of two replicates. $* p<0.05$, and fold change $>2.0$. Statistics were calculated between irrigated and drought treatments for each cultivar using the AudicClaverie method

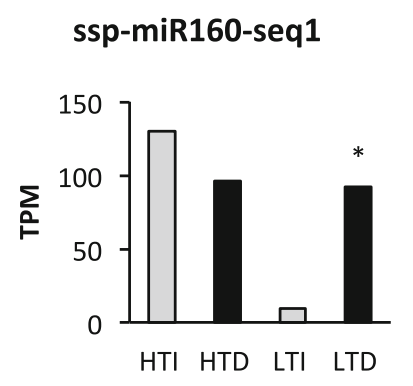

ssp-miR169-seq2

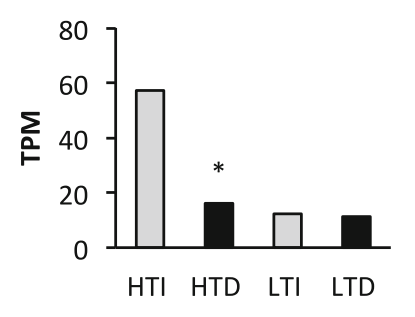

ssp-miR393

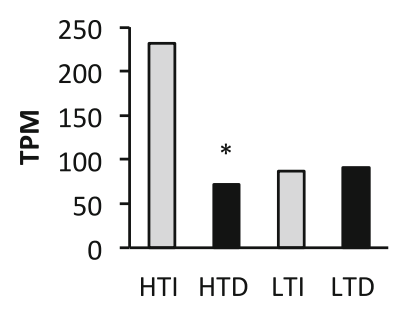

ssp-miR399-seq2

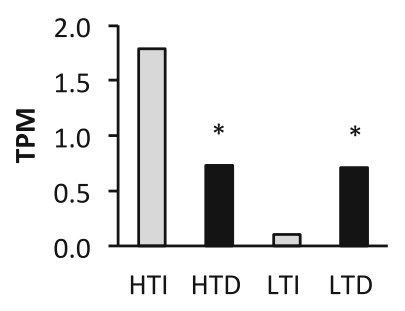

ssp-miR1432

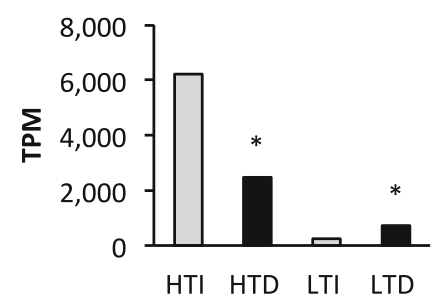

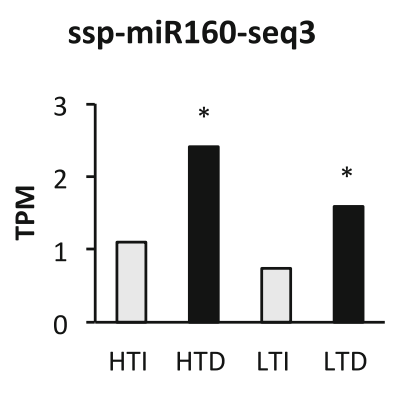

ssp-miR166-seq3

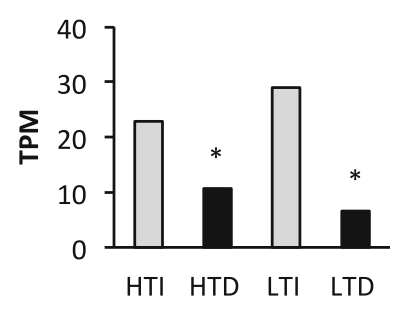

ssp-miR172

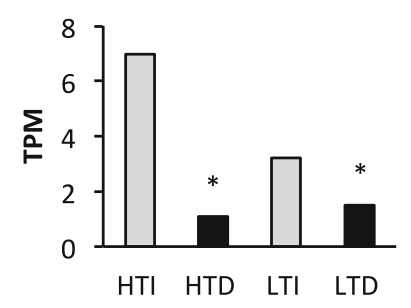

ssp-miR394

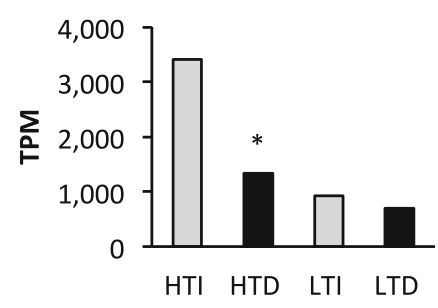

ssp-miR396

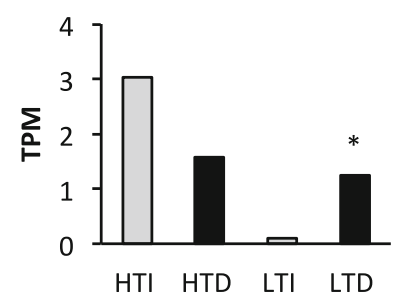

ssp-miR399-seq3
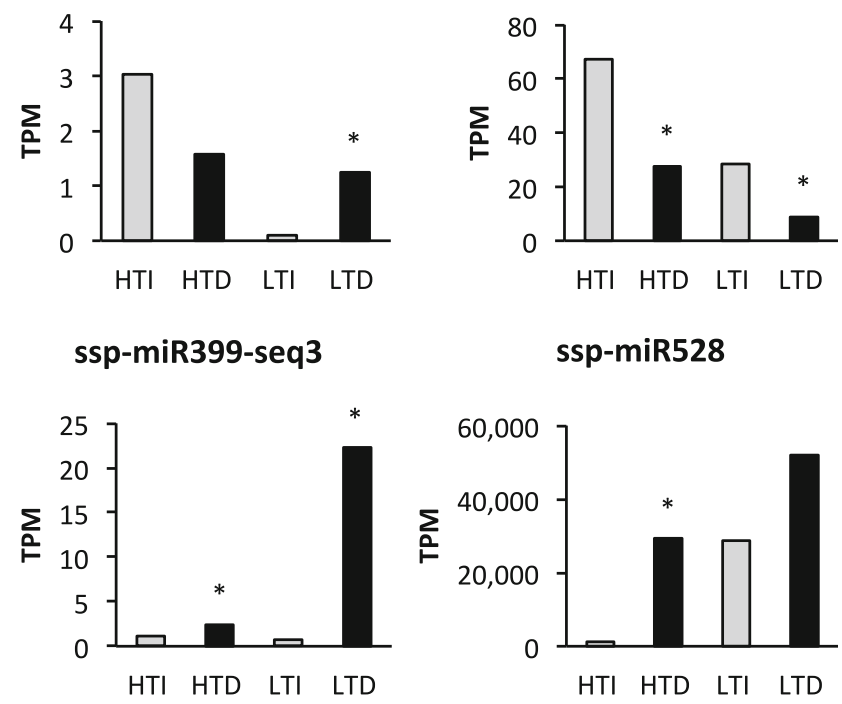

ssp-miR528

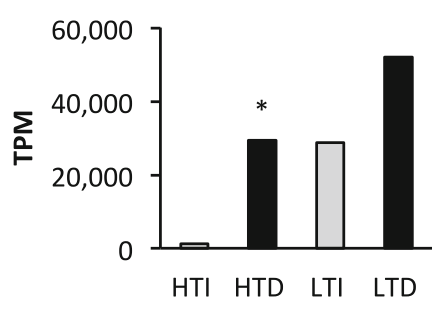

including Saccharum officinarum (sof-miR) and Saccharum spp. (ssp-miR). In this work, we have identified 30 mature miRNA sequences belonging to 18 sugarcane
miRNA families expressed in leaves under drought stress (Table 2), with some precursors identified in SoGI and SUCEST databases. Because miRNA expression could be 


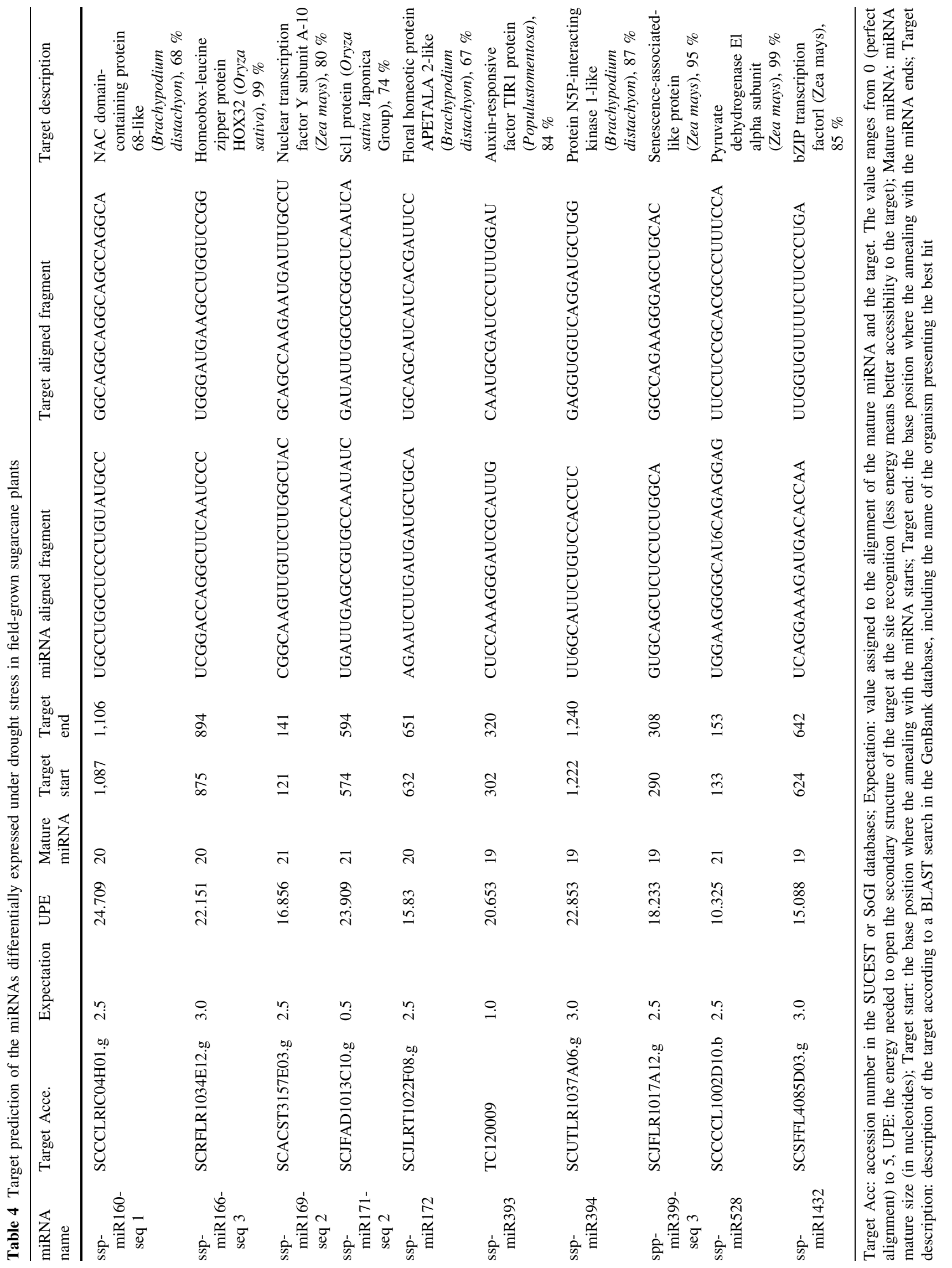



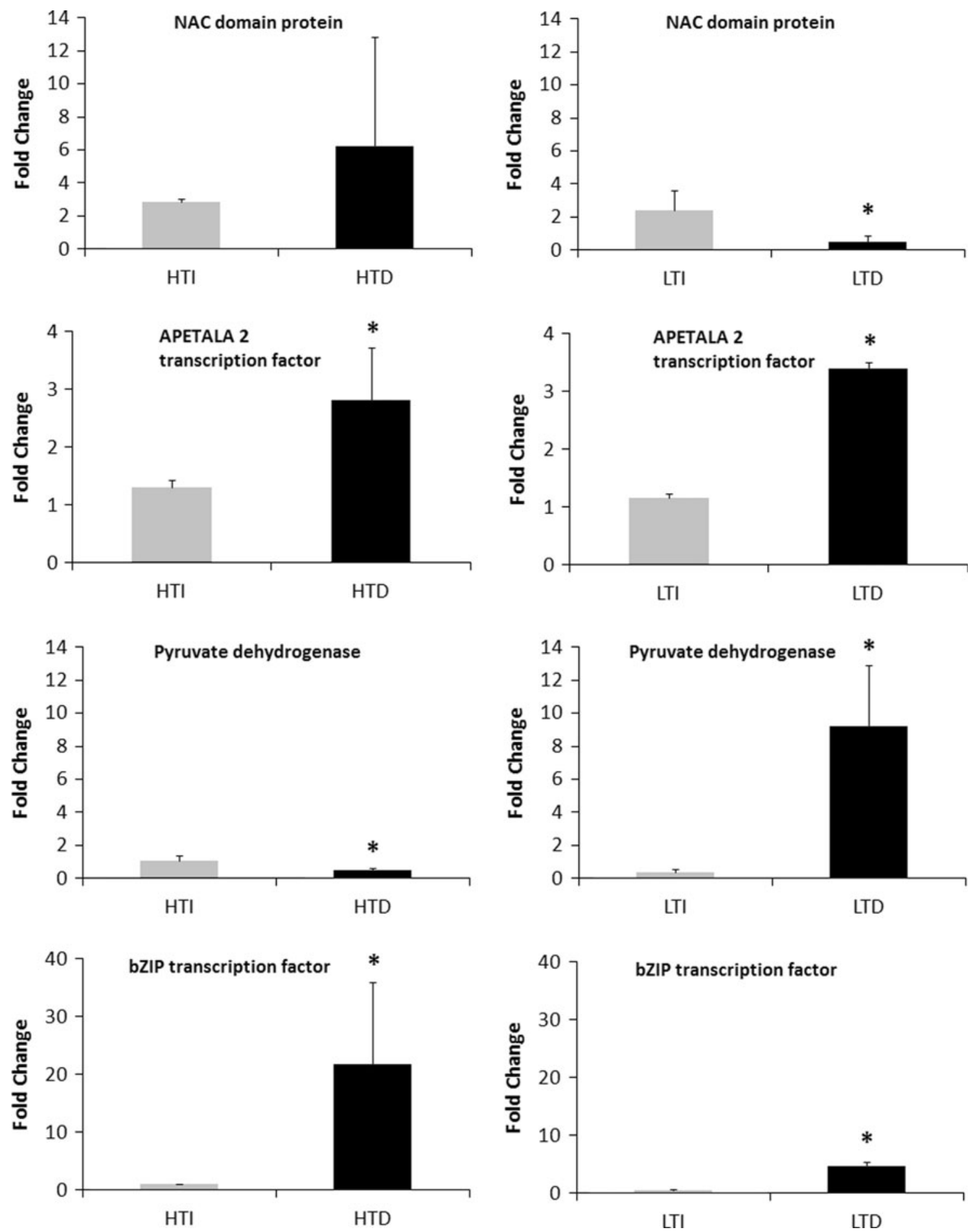

Fig. 4 Expression profile of one of the predicted target genes for four sugarcane miRNAs modulated by drought. The values are expressed as fold changes relative to the irrigated control for each gene. The bars represent the average of the irrigated plants (control, grey bars) and drought-stressed plants (black bars) for RB867515 and

RB855536 after 7 months of stress. Error bars represent the standard deviation $(n=3)$. Statistics were calculated between irrigated and drought-treated plants using a $t$ test. Asterisk indicates the differences between irrigated and drought-stressed plants, with $p \leq 0.05$

specific to tissue, age or development stage, it is expected that the number of sugarcane miRNAs would increase when other tissues were analyzed.

Several new miRNAs, not conserved among the species, have been identified along with their targets, which include genes associated with diverse metabolic pathways and 
Table 5 Expression profiles of selected target genes found for the miRNAs differentially expressed under drought conditions

\begin{tabular}{lllll}
\hline miRNA target & SAS cluster name & Target description & HT7 & LT7 \\
\hline Target miR160-seq 1 & SCCCLR1C04H01.g & NAC domain containing protein 68-like & $\mathbf{0 . 7 4 / 2 . 1 7}$ & $\mathbf{9 . 6 2} * / \mathbf{0 . 1 9} *$ \\
Target miR172 & SCJLRT1022F08.g & Floral homectic protein Apetala2 & $\mathbf{0 . 4} * / \mathbf{2 . 1 6 *}$ \\
Target miR528 & SCCCCL1002D10.b & Pyruvate dehydrogenase E1 alpha subunit & $\mathbf{2 4 . 0 6}^{* / 0.44 *}$ \\
Target miR1432 & SCSFFL4085D03.g & B-ZIP transcription factor & $\mathbf{0 . 4 0}^{*} / \mathbf{2 0 . 1} *$
\end{tabular}

The SAS cluster name and the complete description of each target gene are described in the table. HT: RB867515 (higher drought tolerant plants); LT: RB855536 (lower drought tolerant plants). The expression ratios between drought-stressed and control plants are shown. The first number in each pair indicates miRNA levels and the second indicates the target gene expression. Asterisk indicates ratios where differences in the expression levels in the irrigated and drought-stressed plants are statistically significant $(p<0.05)$. The bold expression ratios indicate that miRNA induction or repression correlates with repression or induction of target genes, respectively. Statistics were calculated between irrigated and drought treatments using a $t$ test

SAS sugarcane assembled sequence, $H T$ higher tolerance cultivar, $L T$ lower tolerance cultvar, 7, 7 months of stress

cellular processes related with the development of resistance to abiotic stresses (Eckardt 2004; Llave et al. 2002; Sunkar and Zhu 2004). Using P. trichocarpa as a model, Lu et al. (2005) confirmed the in silico-predicted targets as genes related to development and/or stress, with putative associated functions as cell wall metabolites important in the regulation of wood development in trees. Although some miRNAs share conserved sequences, most of them exhibit species-specific expression profiles during development, suggesting that conserved miRNAs could have different regulatory roles in different species.

Sugarcane miRNA targets were predicted by psRNAtarget and some of these targets were validated by RT-qPCR (Table 5; Fig. 4). 10 out of 13 drought-modulated miRNAs predicted multiple target genes in the SUCEST database and 1 predicted target gene based on the SoGI database (Table 4, Supplementary Table S2). The majority of the targets encoded transcription factors, as already described for other organisms (Rhoades et al. 2002).

The expression profile of ssp-miR160-seq 1 was dependent on the cultivar and treatment, being significantly induced in the LT cultivar (RB855536) and slightly repressed in the HT cultivar (RB867515). One of the targets for this miRNA is a protein containing a NAC domain. Proteins from this family are known to be induced by diverse abiotic factors (Ditt et al. 2011; Hegedus et al. 2003; Sun et al. 2011). Although we found a high variability in the expression of the target gene in the fieldgrown plants, the ssp-miR160-seq 1 and target gene expression profiles indicated that LT plants repress and have lower levels of the target gene, while the opposite profile was observed in HT plants. Therefore, the gene encoding a NAC protein might play a role in the differences observed between the HT and LT cultivars.

ssp-miR166-seq 3 was repressed in both HT and LT cultivars and targets genes encoding homeobox-leucine zipper proteins, a family of transcription factors found only in plants. Transgenic Arabidopsis plants overexpressing the Helianthus annus Hahb-4 gene presented increased tolerance to water stress (Dezar et al. 2005). The downregulation of ssp-miR166-seq 3 in both cultivars, leading to higher levels of the homeobox-leucine zipper protein, would enhance sugarcane tolerance to drought stress.

Previous studies in rice ( $O$. sativa) showed that some members of the miR169 family were induced by drought (Zhao et al. 2007a) and salt stress (Zhao et al. 2009), while other members of this family were repressed ( $\mathrm{Li}$ et al. 2008; Zhao et al. 2007a). ssp-miR169-seq 2 target genes belong to the Nuclear Factor YA family (NF-Y), a group of transcription factors that have three distinct subunits (NF-YA, NF-YB, and NF-YC) that bind to the CCAAT box (Combier et al. 2006; Li et al. 2008). Nine NF- $Y$ genes expressed in wheat leaves responded to drought stress (Stephenson et al. 2007). The NF-YA genes have been reported to be involved in plant drought resistance, and the overexpression of NF-YA5 and NF-YB1 in Arabidopsis can also provide drought tolerance (Li et al. 2008; Nelson et al. 2007). Another interesting putative target encodes a glutathione S-transferase. These enzymes are well known for their role in protecting plants from oxidative stress. In sugarcane, we found that ssp-miR169 was downregulated in the HT cultivar (RB867515) during water deficit (Fig. 3; Table 3), suggesting that its target genes were upregulated. This upregulation suggests increased expression of $N F-Y$ genes and lower oxidative stress in the HT plants, implicating these genes in sugarcane drought tolerance. However, ssp-miR169-seq 2 expression in LT plants was unchanged. Moreover, ssp-mR169-seq 2 levels were similar to what was observed in HT plants under drought stress, indicating that the target genes would be expressed at similar levels in both sugarcane cultivars during drought stress. 
ssp-miR171-seq 2 was downregulated after 7 months of stress in both cultivars. A target gene for this miRNA encodes a protein with similarity to members of the GRAS/ SCL family (Table 4). Members of this family include transcription factors that participate in diverse plant growth pathways and respond to stress, namely abiotic stress. The Scarecrow-like genes (SCL) belong to the GRAS multigenic family that are named for the following three loci: Gibberellic-acid insensitive (GAI), the GAI repressor (RGA) and the Scarecrow (SCR) (Pysh et al. 1999). Two of the members of the GRAS family, GAI and RGA, participate in the Gibberellic acid (GA) signal transduction pathway. The rice SLR1 was identified as GAI orthologue and is involved in the GA pathway in maize, grape, wheat, and barley (Hynes et al. 2003). In poplars, the PeSCL7 gene was induced by drought and salt stress, and conferred tolerance to these stressors when overexpressed in Arabidopsis (Ma et al. 2010). The downregulation of sspmiR171-seq 2, by increasing the levels of the sugarcane $S C L$ target gene, which may activate other genes, might contribute to an increase in drought tolerance in both cultivars.

We found that ssp-miR172 was downregulated in both cultivars under drought conditions, with a major change in the HT cultivar (RB867515) (Fig. 3; Table 3). There are three transcription factors among the predicted target genes for this particular miRNA. One of them belongs to the APETALA2 family of transcription factors (Table 4) and was induced in both cultivars (Fig. 4; Table 5). In rice, osamiR172 was downregulated by water deficit stress (Zhou et al. 2010). The APETALA2 family is one of the largest families of transcription factors in Arabidopsis, with 145 loci (Sakuma et al. 2002). Some members, such as the $D R E B$ genes, are involved in plant responses to drought and salt stress (Krishnaswamy et al. 2011). Therefore, sspmiR172 may increase the expression of transcription factors that activate plant responses to drought stress.

ssp-miR393 presented no changes in the LT cultivar and was significantly repressed in the HT cultivar under drought (Fig. 3). One of the putative targets encodes a protein similar to TIR1, an auxin receptor in A. thaliana (Table 4). TIR1 recognizes 3-indole-acetic acid (AIA) and promotes the degradation of the Aux/AIA repressor by a protein ubiquitin ligase that binds to a conserved area of the repressor and allows transcription of auxin-regulated genes (Dharmasiri et al. 2005). Recently, the expression of TIRI was associated with the response of $A$. thaliana roots to inorganic phosphate (Pi) (Perez-Torres et al. 2008). Our data suggest that in HT cultivars, ssp-miR393 is involved in the regulation of genes that modulate auxin activity in leaves during drought stress.

ssp-miR394 was downregulated under drought stress in the HT sugarcane cultivar and significantly upregulated in the LT cultivar, showing a different response between these genotypes. Among the predicted targets of ssp-miR394 is a gene encoding the protein NSP-interacting kinase (NIK) (Table 4). NIK belongs to a receptor-like serine/threonine kinase subfamily, the members of which contain five leucine-rich repeats that are involved in plant development and the response to biotic stresses, namely viral stress (Santos et al. 2010). This is the first study relating this kinase with drought response.

ssp-miR399-seq 3 was induced in both cultivars, and had significantly higher levels in the LT cultivar. A putative target of ssp-miR-399-seq 3 encodes a senescenceassociated protein (Table 4). Leaf senescence is a symptom of water deficit and transgenic tobacco plants with delayed leaf senescence had increased tolerance to drought (Rivero et al. 2010). The upregulation of ssp-miR399-seq 3, by decreasing the levels of the senescence-associated protein, might contribute to an increase in drought tolerance in sugarcane.

ssp-miR528 has several putative targets, and we have evaluated by RT-qPCR the gene encoding a pyruvate dehydrogenase. This enzyme is part of a pyruvate dehydrogenase complex (PDHc) that plays a pivotal role in cell metabolism, catalyzing the oxidative decarboxylation of pyruvate and the subsequent acetylation of coenzyme A to acetyl-CoA (Gutowski and Lienhard 1976; Nemeria et al. 2001). Pyruvate dehydrogenase links the glycolysis metabolic pathway to the citric acid cycle, where the acetylCoA is used to carry out cellular respiration. In this work, both sugarcane cultivars showed an increase in ssp-miR528 expression under drought conditions, where the HT cultivar has a higher increase than the LT cultivar. This expression increase may prevent loss of $\mathrm{CO}_{2}$ to the atmosphere by leaf respiration, leading to better control of carbon balance during drought stress (Chaves et al. 2002, 2009; Flexas et al. 2006).

The ssp-miR1432 miRNA was downregulated under drought stress in HT plants and induced in the LT cultivar. This miRNA also has several putative targets, and we have evaluated one target encoding a bZIP transcription factor that has been shown to confer stress tolerance to plants (Golldack et al. 2011). The ssp-miR1432 expression pattern indicates that HT plants adjust their transcriptome to increase the bZIP factor, which may activate the transcription of drought-related genes.

Recently, we have evaluated sugarcane miRNAs that had their expression patterns modulated by drought in 3-monthold plants grown in a greenhouse (Ferreira et al. 2012). Seven drought-responsive miRNAs were identified in these plants: ssp-miR164, ssp-miR393, ssp-miR394, ssp-miR397, ssp-miR399-seq 1, ssp-miR528, and ssp-miR1432. Interestingly, four miRNAs (ssp-miR393, ssp-miR394, sspmiR528, and spp-miR1432) were modulated by drought in 
both greenhouse and field experiments, but only sspmiR528 (up-regulated) and ssp-miR1432 (down-regulated) had similar expression patterns in both experiments, and only in the HT cultivar. In another work using sugarcane 3-month-old plants, roots exposed to drought stress for $24 \mathrm{~h}$ presented five miRNAs that were differentially expressed (Thiebaut et al. 2012). None of them were observed in the leaves from plants grown under field conditions. These findings indicate that sugarcane response to drought is affected by plant age and organ, and also by the experimental conditions.

In summary, we have studied two cultivars grown in the field for 7 months that differ in drought tolerance, with or without irrigation. Field experiments provide more reliable information on the effect of stress on plants in a natural environment. We have shown that the expression patterns of several miRNAs are modulated by drought in the field and that some may play a significant role in the higher drought tolerance observed in the RB867515 cultivar. Finally, we showed evidence that miRNA expression profiles may vary according to the genetic background from the distinct sugarcane cultivars.

Acknowledgments AG received a fellowship from National Council for Scientific and Technological Development (CNPq); THF and RSM received a fellowship from São Paulo Research Foundation (FAPESP); GMS, RJMCN, LE and MM were recipients of Research Productivity Fellowships from CNPq. This research was developed as part of a research network involving grants 2008/5798-6 and 2008/57908-6 from FAPESP, 574002/2008-1 and 552802/2007-7 from CNPq and 815/07 from Brazilian Innovation Agency (FINEP).

Open Access This article is distributed under the terms of the Creative Commons Attribution License which permits any use, distribution, and reproduction in any medium, provided the original author(s) and the source are credited.

\section{References}

Agarwal PK, Agarwal P, Reddy MK, Sopory SK (2006) Role of DREB transcription factors in abiotic and biotic stress tolerance in plants. Plant Cell Rep 25:1263-1274

Audic S, Claverie JM (1997) The significance of digital gene expression profiles. Genome Res 7:986-995

Axtell MJ (2008) Evolution of microRNAs and their targets: are all microRNAs biologically relevant? BBA Gene Regul Mech 1779:725-734

Axtell MJ, Bartel DP (2005) Antiquity of microRNAs and their targets in land plants. Plant Cell 17:1658-1673

Bartel DP (2004) MicroRNAs: genomics, biogenesis, mechanism, and function. Cell 116:281-297

Bentley DR (2006) Whole-genome re-sequencing. Curr Opin Genet Dev 16:545-552

Boyer RS (1982) Plant productivity and environment. Science 218:443-448

Carrington JC, Ambros V (2003) Role of microRNAs in plant and animal development. Science 301:336-338

Chapman EJ, Carrington JC (2007) Specialization and evolution of endogenous small RNA pathways. Nat Rev Genet 8:884-896
Chaves MM, Pereira JS, Maroco J, Rodrigues ML, Ricardo CP, Osorio ML, Carvalho I, Faria T, Pinheiro C (2002) How plants cope with water stress in the field. Photosynthesis and growth. Ann Bot 89:907-916

Chaves MM, Flexas J, Pinheiro C (2009) Photosynthesis under drought and salt stress: regulation mechanisms from whole plant to cell. Ann Bot 103:551-560

Chen CF, Ridzon DA, Broomer AJ, Zhou ZH, Lee DH, Nguyen JT, Barbisin M, Xu NL, Mahuvakar VR, Andersen MR, Lao KQ, Livak KJ, Guegler KJ (2005) Real-time quantification of microRNAs by stem-loop RT-PCR. Nucleic Acids Res 33:e179

Combier JP, Frugier F, de Billy F, Boualem A, El-Yahyaoui F, Moreau S, Vernie T, Ott T, Gamas P, Crespi M, Niebel A (2006) MtHAP2-1 is a key transcriptional regulator of symbiotic nodule development regulated by microRNA169 in Medicago truncatula. Genes Dev 20:3084-3088

Dai X, Zhao PX (2011) psRNATarget: a plant small RNA target analysis server. Nucleic Acids Res 39:W155-W159

Dezar CA, Gago GM, Gonzalez DH, Chan RL (2005) Hahb-4, a sunflower homeobox-leucine zipper gene, is a developmental regulator and confers drought tolerance to Arabidopsis thaliana plants. Transgenic Res 14:429-440

Dharmasiri N, Dharmasiri S, Estelle M (2005) The F-box protein TIR1 is an auxin receptor. Nature 435:441-445

Ditt RF, Gentile A, Tavares RG, Camargo SR, Fernandez JH, da Silva MJ, Menossi M (2011) Analysis of the stress-inducible transcription factor SsNAC23 in sugarcane plants. Sci Agr 68:454461

Eckardt NA (2004) Small RNA on the move. Plant Cell 16:1951-1954

Fahlgren N, Carrington JC (2010) miRNA target prediction in plants. Methods Mol Biol 592:51-57

Ferreira TH, Gentile A, Vilela RD, Costa GGL, Dias LI, Endres L, Menossi M (2012) microRNAs associated with drought response in the bioenergy crop sugarcane (Saccharum spp.). PLoS One 7:e46703

Flexas J, Bota J, Galmes J, Medrano H, Ribas-Carbo M (2006) Keeping a positive carbon balance under adverse conditions: responses of photosynthesis and respiration to water stress. Physiol Plantarum 127:343-352

Floyd SK, Bowman JL (2004) Gene regulation: ancient microRNA target sequences in plants. Nature 428:485-486

Golldack D, Luking I, Yang O (2011) Plant tolerance to drought and salinity: stress regulating transcription factors and their functional significance in the cellular transcriptional network. Plant Cell Rep 30:1383-1391

Gustafson AM, Allen E, Givan S, Smith D, Carrington JC, Kasschau KD (2005) ASRP: the Arabidopsis small RNA project database. Nucleic Acids Res 33:D637-D640

Gutowski JA, Lienhard GE (1976) Transition-state analogs for thiamin pyrophosphate-dependent enzymes. J Biol Chem 251:2863-2866

Hegedus D, Yu M, Baldwin D, Gruber M, Sharpe A, Parkin I, Whitwill S, Lydiate D (2003) Molecular characterization of Brassica napus NAC domain transcriptional activators induced in response to biotic and abiotic stress. Plant Mol Biol 53:383-397

Hoen PA, Ariyurek Y, Thygesen HH, Vreugdenhil E, Vossen RH, De Menezes RX, Boer JM, Van Ommen GJ, Den Dunnen JT (2008) Deep sequencing-based expression analysis shows major advances in robustness, resolution and inter-lab portability over five microarray platforms. Nucleic Acids Res 36:e141

Hofacker IL (2003) Vienna RNA secondary structure server. Nucleic Acids Res 31:3429-3431

Hynes LW, Peng J, Richards DE, Harberd NP (2003) Transgenic expression of the Arabidopsis DELLA proteins GAI and gai 
confers altered gibberellin response in tobacco. Transgenic Res 12:707-714

Inman-Bamber NG, Smith DM (2005) Water relations in sugarcane and response to water deficits. Field Crop Res 92:185-202

Jian XY, Zhang L, Li GL, Zhang L, Wang XJ, Cao XF, Fang XH, Chen $F$ (2010) Identification of novel stress-regulated microRNAs from Oryza sativa L. Genomics 95:47-55

Jones-Rhoades MW, Bartel DP (2004) Computational identification of plant microRNAs and their targets, including a stress-induced miRNA. Mol Cell 14:787-799

Jones-Rhoades MW, Bartel DP, Bartel B (2006) MicroRNAS and their regulatory roles in plants. Annu Rev Plant Biol 57:19-53

Kidner C, Timmermans M (2006) In situ hybridization as a tool to study the role of microRNAs in plant development. Methods Mol Biol 342:159-179

Krishnaswamy S, Verma S, Rahman MH, Kav NN (2011) Functional characterization of four APETALA2-family genes (RAP2.6, RAP2.6L, DREB19 and DREB26) in Arabidopsis. Plant Mol Biol 75:107-127

Li WX, Oono Y, Zhu J, He XJ, Wu JM, Iida K, Lu XY, Cui X, Jin H, Zhu JK (2008) The Arabidopsis NFYA5 transcription factor is regulated transcriptionally and posttranscriptionally to promote drought resistance. Plant Cell 20:2238-2251

Llave C, Kasschau KD, Rector MA, Carrington JC (2002) Endogenous and silencing-associated small RNAs in plants. Plant Cell 14:1605-1619

Lu S, Sun YH, Shi R, Clark C, Li L, Chiang VL (2005) Novel and mechanical stress-responsive MicroRNAs in Populus trichocar$p a$ that are absent from Arabidopsis. Plant Cell 17:2186-2203

Ma HS, Liang D, Shuai P, Xia XL, Yin WL (2010) The salt- and droughtinducible poplar GRAS protein SCL7 confers salt and drought tolerance in Arabidopsis thaliana. J Exp Bot 61:4011-4019

Matts J, Jagadeeswaran G, Roe BA, Sunkar R (2010) Identification of microRNAs and their targets in switchgrass, a model biofuel plant species. J Plant Physiol 167:896-904

Maybank J, Bonsal BR, Jones K, Lawford RG, O'Brien EG et al (1995) Drought as a natural disaster. Atmos Ocean 33:195-222

Meyers BC, Axtell MJ, Bartel B, Bartel DP, Baulcombe D, Bowman JL, Cao X, Carrington JC, Chen X, Green PJ, Griffiths-Jones S, Jacobsen SE, Mallory AC, Martienssen RA, Poethig RS, Qi Y, Vaucheret H, Voinnet O, Watanabe Y, Weigel D, Zhu JK (2008) Criteria for annotation of plant MicroRNAs. Plant Cell 20:3186-3190

Molnar A, Schwach F, Studholme DJ, Thuenemann EC, Baulcombe DC (2007) miRNAs control gene expression in the single-cell alga Chlamydomonas reinhardtii. Nature 447:1126-1129

Nelson DE, Repetti PP, Adams TR, Creelman RA, Wu J, Warner DC, Anstrom DC, Bensen RJ, Castiglioni PP, Donnarummo MG, Hinchey BS, Kumimoto RW, Maszle DR, Canales RD, Krolikowski KA, Dotson SB, Gutterson N, Ratcliffe OJ, Heard JE (2007) Plant nuclear factor Y (NF-Y) B subunits confer drought tolerance and lead to improved corn yields on water-limited acres. Proc Natl Acad Sci USA 104:16450-16455

Nemeria N, Yan Y, Zhang Z, Brown AM, Arjunan P, Furey W, Guest JR, Jordan F (2001) Inhibition of the Escherichia coli pyruvate dehydrogenase complex E1 subunit and its tyrosine 177 variants by thiamin 2-thiazolone and thiamin 2-thiothiazolone diphosphates-evidence for reversible tight-binding inhibition. J Biol Chem 276:45969-45978

Pabinger S, Thallinger GG, Snajder R, Eichhorn H, Rader R, Trajanoski Z (2009) QPCR: application for real-time PCR data management and analysis. BMC Bioinform 10:268

Pantaleo V, Szittya G, Moxon S, Miozzi L, Moulton V, Dalmay T, Burgyan J (2010) Identification of grapevine microRNAs and their targets using high-throughput sequencing and degradome analysis. Plant J 62:960-976
Papini-Terzi FS, Rocha FR, Vencio RZN, Oliveira KC, Felix JD, Vicentini R, Rocha CD, Simoes ACQ, Ulian EC, Di Mauro SMZ, Da Silva AM, Pereira CAD, Menossi M, Souza GM (2005) Transcription profiling of signal transduction-related genes in sugarcane tissues. DNA Res 12:27-38

Perez-Torres CA, Lopez-Bucio J, Cruz-Ramirez A, Ibarra-Laclette E, Dharmasiri S, Estelle M, Herrera-Estrella L (2008) Phosphate availability alters lateral root development in Arabidopsis by modulating auxin sensitivity via a mechanism involving the TIR1 auxin receptor. Plant Cell 20:3258-3272

Pysh LD, Wysocka-Diller JW, Camilleri C, Bouchez D, Benfey PN (1999) The GRAS gene family in Arabidopsis: sequence characterization and basic expression analysis of the Scarecrow-like genes. Plant J 18:111-119

Reinhart BJ, Weinstein EG, Rhoades MW, Bartel B, Bartel DP (2002) MicroRNAs in plants. Genes Dev 16:1616-1626

Rhoades MW, Reinhart BJ, Lim LP, Burge CB, Bartel B, Bartel DP (2002) Prediction of plant microRNA targets. Cell 110:513-520

Rivero RM, Gimeno J, Van Deynze A, Walia H, Blumwald E (2010) Enhanced cytokinin synthesis in tobacco plants expressing PSARK: IPT prevents the degradation of photosynthetic protein complexes during drought. Plant Cell Physiol 51:1929-1941

Rocha FR, Papini-Terzi FS, Nishiyama MY, Vencio RZN, Vicentini R, Duarte RDC, de Rosa VE, Vinagre F, Barsalobres C, Medeiros AH, Rodrigues FA, Ulian EC, Zingaretti SM, Galbiatti JA, Almeida RS, Figueira AVO, Hemerly AS, Silva-Filho MC, Menossi M, Souza GM (2007) Signal transduction-related responses to phytohormones and environmental challenges in sugarcane. BMC Genomics 8:71

Ruan MB, Zhao YT, Meng ZH, Wang XJ, Yang WC (2009) Conserved miRNA analysis in Gossypium hirsutum through small RNA sequencing. Genomics 94:263-268

Ruby JG, Jan C, Player C, Axtell MJ, Lee W, Nusbaum C, Ge H, Bartel DP (2006) Large-scale sequencing reveals 21U-RNAs and additional microRNAs and endogenous siRNAs in C. elegans. Cell 127:1193-1207

Sakuma Y, Liu Q, Dubouzet JG, Abe H, Shinozaki K, YamaguchiShinozaki K (2002) DNA-binding specificity of the ERF/AP2 domain of Arabidopsis DREBs, transcription factors involved in dehydration- and cold-inducible gene expression. Biochem Biophys Res Commun 290:998-1009

Santos D (2010) Distribuição do sistema radicular e produtividade de cana-de-açúcar (Saccharum ssp.) fertirrigada por gotejamento subsuperficial. Dissertation, Universidade Estadual Paulista "Júlio de Mesquita Filho"

Santos AA, Lopes KV, Apfata JA, Fontes EP (2010) NSP-interacting kinase, NIK: a transducer of plant defence signalling. J Exp Bot 61:3839-3845

Shendure J, Porreca GJ, Reppas NB, Lin X, McCutcheon JP, Rosenbaum AM, Wang MD, Zhang K, Mitra RD, Church GM (2005) Accurate multiplex polony sequencing of an evolved bacterial genome. Science 309:1728-1732

Stephenson TJ, McIntyre CL, Collet C, Xue GP (2007) Genome-wide identification and expression analysis of the NF-Y family of transcription factors in Triticum aestivum. Plant Mol Biol 65:77-92

Sun H, Huang X, Xu X, Lan H, Huang J, Zhang H-S (2011) ENAC1, a NAC transcription factor, is an early and transient response regulator induced by abiotic stress in rice (Oryza sativa L.). Mol Biotechnol 52:1-10

Sunkar R, Zhu JK (2004) Novel and stress-regulated microRNAs and other small RNAs from Arabidopsis. Plant Cell 16:2001-2019

Thiebaut F, Grativol C, Carnavale-Bottino M, Rojas CA, Tanurdzic M, Farinelli L, Martienssen RA, Hemerly AS, Ferreira PC (2012) Computational identification and analysis of novel sugarcane microRNAs. BMC Genomics 13:290 
Unver T, Budak H (2009) Conserved microRNAs and their targets in model grass species Brachypodium distachyon. Planta 230: 659-669

Varkonyi-Gasic E, Wu RM, Wood M, Walton EF, Hellens RP (2007) Protocol: a highly sensitive RT-PCR method for detection and quantification of microRNAs. Plant Methods 3:12

Vasconcelos ACM, Casagrande AA, Perecin D, Jorge LAC, Landell MGA (2003) Avaliação do sistema radicular da cana-de-açúcar por diferentes métodos. R Bras Ci Solo 27:849-858

Vettore AL, da Silva FR, Kemper EL, Arruda P (2001) The libraries that made SUCEST. Genet Mol Biol 24:1-7

Voinnet O (2009) Origin, biogenesis, and activity of plant microRNAs. Cell 136:669-687

Wei B, Cai T, Zhang RZ, Li AL, Huo NX, Li S, Gu YQ, Vogel J, Jia JZ, Qi YJ, Mao L (2009) Novel microRNAs uncovered by deep sequencing of small RNA transcriptomes in bread wheat (Triticum aestivum L.) and Brachypodium distachyon (L.) Beauv. Funct Integr Genomic 9:499-511

Wu L, Zhang QQ, Zhou HY, Ni FR, Wu XY, Qi YJ (2009) Rice microRNA effector complexes and targets. Plant Cell 21: 3421-3435

Xin M, Wang Y, Yao Y, Xie C, Peng H, Ni Z, Sun Q (2010) Diverse set of microRNAs are responsive to powdery mildew infection and heat stress in wheat (Triticum aestivum L.). BMC Plant Biol 10:123

Yang XZ, Li L (2011) miRDeep-P: a computational tool for analyzing the microRNA transcriptome in plants. Bioinformatics 27: 2614-2615

Yang XZ, Zhang HY, Li L (2011) Global analysis of gene-level microRNA expression in Arabidopsis using deep sequencing data. Genomics 98:40-46
Zanca AS, Vicentini R, Ortiz-Morea FA, Del Bem LEV, da Silva MJ, Vincentz M, Nogueira FTS (2010) Identification and expression analysis of microRNAs and targets in the biofuel crop sugarcane. BMC Plant Biol 10:260

Zhang J, Xu Y, Huan Q, Chong K (2009a) Deep sequencing of Brachypodium small RNAs at the global genome level identifies microRNAs involved in cold stress response. BMC Genomics 10:449

Zhang LF, Chia JM, Kumari S, Stein JC, Liu ZJ, Narechania A, Maher CA, Guill K, McMullen MD, Ware D (2009b) A genomewide characterization of microRNA genes in maize. Plos Genet 5:e1000716

Zhao B, Liang R, Ge L, Li W, Xiao H, Lin H, Ruan K, Jin Y (2007a) Identification of drought-induced microRNAs in rice. Biochem Biophys Res Commun 354:585-590

Zhao BT, Liang RQ, Ge LF, Li W, Xiao HS, Lin HX, Ruan KC, Jin YX (2007b) Identification of drought-induced microRNAs in rice. Biochem Biophy Res Comm 354:585-590

Zhao T, Li G, Mi S, Li S, Hannon GJ, Wang XJ, Qi Y (2007c) A complex system of small RNAs in the unicellular green alga Chlamydomonas reinhardtii. Genes Dev 21:1190-1203

Zhao B, Ge L, Liang R, Li W, Ruan K, Lin H, Jin Y (2009) Members of miR-169 family are induced by high salinity and transiently inhibit the NF-YA transcription factor. BMC Mol Biol 10:29

Zhao CZ, Xia H, Frazier TP, Yao YY, Bi YP, Li AQ, Li MJ, Li CS, Zhang BH, Wang XJ (2010) Deep sequencing identifies novel and conserved microRNAs in peanuts (Arachis hypogaea L.). BMC Plant Biol 10:3

Zhou L, Liu Y, Liu Z, Kong D, Duan M, Luo L (2010) Genome-wide identification and analysis of drought-responsive microRNAs in Oryza sativa. J Exp Bot 61:4157-4168 